\section{CONDICIONES DE FORMA Y DESEMPEÑO ENERGÉTICO DE VIVIENDAS UNIFAMILIARES EN EL CENTRO-SUR DE CHILE}

Rodrigo García Álvarado², Alex González ${ }^{3}$

\section{FORM CONDITIONS AND ENERGY PERFORMANCE OF SINGLE-FAMILY HOUSING IN CENTRAL-SOUTH CHILE ${ }^{1}$}

Rodrigo García Alvarado², Alex González ${ }^{3}$

\section{Resumen}

El sector residencial presenta un creciente consumo energético, y las condiciones de forma han sido sugeridas como uno de los aspectos más incidentes en el comportamiento ambiental de las edificaciones. La zona centro-sur de Chile presenta un predominio de viviendas unifamiliares con volumetrías independientes, que podrían tener una influencia relevante en su desempeño energético. Con el fin de esclarecer la incidencia de características formales en el comportamiento ambiental de esta tipología este artículo expone una revisión de varios estudios y de las principales condiciones de las viviendas en la zona, y luego un análisis

\section{Abstract}

The energy consumption of the residential sector has been increasing and the formconditions have been suggested as one of the critical aspects in the environmental behavior of dwellings. In centralsouth Chile, there is a predominance of single-family housing with independent volumetrics, which may have a considerable effect on their energy performance. In order to clarify the effect of formal characteristics on the environmental behavior of this typology, the paper reviewsseveral studies and the main conditions of dwellings in the area, followed by an analysis based on energy simulations of a model with different configurations with the 
mediante simulaciones energéticas de un modelo de diferentes configuraciones con la misma superficie y características constructivas. Identificando una influencia relevante de la variación, contigüidad y altura en la demanda energética, con una escasa incidencia de la orientación solar. Al final se sugiere un factor de regulación, correspondiente a la superficie de muros expuestos, lo que expresa su relevancia formal y material, y puede ser implementado para fomentar la reducción del gasto energético y mejoramiento ambiental de las viviendas en la zona.

\section{PALABRAS CLAVE: EFICIENCIA ENERGÉTICA, SIMULACIÓN AMBIENTAL, VIVIENDA UNIFAMILIAR, CHILE}

Fecha de recepción: 08.03.2013

Fecha de aceptación: 17.01.2014

1 Proyecto Fondecyt 1120165 "Evaluación de Modelos de Simulación Ambiental de Viviendas de la zona Centro-Sur de Chile" (2012-2015), y MEL-Conicyt 8110003 "Diseño integrado para la reconstrucción de viviendas energéticamente eficientes" (2011-2013).

2 Chile. Arquitecto, Pontificia Universidad Católica de Chile. Master en Arquitectura, Universidad Politécnica de Madrid, España. Doctor en Arquitectura, Universidad Politécnica de Cataluña, España. Académico del Departamento de Diseño y Teoría de la Arquitectura y Director del Doctorado en Arquitectura de la Universidad del Bío-Bío. Correo electrónico: rGarcía@ubiobio.cl

3 Chile. Investigador del Centro de Investigación en Tecnologías de la Construcción de la Universidad del Bío-Bío. Correo electrónico: alex.gonzalez.caceres@gmail.com same surface and constructional properties. The analysis results enable the identification of an important influence on the variation, contiguity and height of energy demand, with low impact of solar orientation. The conclusions suggest a regulatory factor corresponding to the surface of exposed walls, which is expressed on their formal and material importance; such a factor may be implemented to promote the energy consumption reduction and the environmental improvement of dwellings in the area.

\section{KEYWORDS: ENERGY EFFICIENCY, ENVIRONMENTAL SIMULATION, SINGLE-FAMILY HOUSING, CHILE}

Received: 08.03.2013
Accepted: 17.01.2014

1 Fondecytproject 1120165 "Evaluation of environmental simulation models of housing in Central-South Chile" (2012-2015) and MEL-Conicyt 8110003 “Integral design for the reconstruction of energy efficient housing" (2011-2013).

2 Chile. Architect, Pontifical Catholic University of Chile.MA in Architecture, Technical University of Madrid, Spain.PhD in Architecture, Technical University of Catalonia, Spain.Academic, Department of Design and Theory of Architecture and Director of the Doctoral Program in Architecture, University of Bio-Bio. Email: rgarcia@ubiobio.cl

3 Chile. Researcher at the Research Center on Construction Technologies, University of Bio-Bio. Email: alex.gonzalez.caceres@ gmail.com 


\section{Introducción}

El desempeño energético de las viviendas es una preocupación creciente por su relevante participación en el consumo general (un 22\% en Chile el año 2011) $)^{4}$, que proviene mayormente de fuentes combustibles foráneas y no-renovables, por tanto con un alto impacto ecológico. Además de su incidencia en la calidad ambiental y el confort interior de las viviendas, con un escalamiento de precios que debilita mantener condiciones adecuadas, especialmente en sectores sociales más vulnerables. Lo que ha motivado múltiples estudios y acciones para mejorar la eficiencia energética de las viviendas, la mayor parte dedicadas a la materialidad de la envolvente y el rendimiento de los equipos domésticos. Sin embargo varios autores sugieren que también la forma general de los edificios posee una alta relevancia en su comportamiento ambiental ${ }^{5}$. Lo que es especialmente importante en el sector residencial, debido a que en los países en desarrollo, gran parte de las viviendas son construcciones independientes. Por lo que su volumetría podría tener una implicancia significativa en el desempeño. Además que condicionan directamente la magnitud de la envolvente y la dimensión de los equipos aplicados. Por ende, una clarificación de las condiciones formales, permite también optimizar

4 MEC Ministerio de Energía, 2012.

5 Olgyay, 1998; Serra y Coch, 1995; Ruano, 2008. las restantes acciones de mejoramiento. Sin embargo, se desconocen claramente las características geométricas incidentes en las distintas tipologías y áreas geográficas. Este trabajo analiza las condiciones formales predominantes en las viviendas del centro-sur de Chile y su relación con el comportamiento energético, revisando estudios residenciales y efectuando simulaciones paramétricas de una misma tipología constructiva. Para identificar características relevantes que pueden sugerir estrategias de diseño de viviendas más efectivas energéticamente y de mejor calidad ambiental.

La relación de condiciones formales de los edificios con su comportamiento ambiental, ha sido mencionada desde los inicios de las preocupaciones de sostenibilidad en arquitectura, sugiriendo influencias de la volumetría de las construcciones en la absorción solar, pérdidas térmicas o ventilación natural. Planteando en especial una reducción de las demandas energéticas de las edificaciones según las proporciones dimensionales de su forma exterior, pero sin acordar consideraciones específicas. El destacado especialista Victor Olgyay, quien publicó uno de los primeros manuales de arquitectura bioclimática ${ }^{6}$, lo planteaba como la razón entre los lados horizontales, recomendando para las viviendas, formas alargadas con distintas proporciones según las zonas climáticas y con orientaciones

6 Olgyay, 1998, p.86-91. 
preferentes. El conocido manual Neufert ${ }^{7}$, a partir de la norma alemana de 1984, plantea un coeficiente general entre las superficies externas y el volumen del edificio relacionado con la transmitancia térmica. Serra y $\mathrm{Coch}^{8}$, prestigiosos especialistas en el ámbito hispanoamericano, proponen distintos indicadores geométricos de compacidad, porosidad, esbeltez, asentamiento, adosamiento, pesadez, perforación, transparencia, aislamiento y tersura, con diversas repercusiones lumínicas, acústicas y climáticas en los edificios. El manual denominado "Vitrubio Ecológico" menciona una alta incidencia de la superficie expuesta en el consumo de energía, proponiendo formas alargadas este-oeste, pero sin indicar relaciones particulares.

Estas condiciones se suelen expresar como el "factor de forma", concepto que se aplica en diversas áreas (en cálculos de masa forestal o la sismicidad de los edificios), y particularmente en termodinámica para expresar la potencialidad de radiación de calor de un cuerpo homogéneo, según la proporción entre la superficie externa y el volumen global $^{10}$. En edificación esta relación se ha interpretado en correspondencia directa con el requerimiento energético. Es decir, que un valor proporcional menor, otorgado por formas mayores y más simples presentaría una menor demanda (en

\footnotetext{
Neufert, 1995, p.111.

Serra y Coch, 1995, p. 240-260.

Ruano, 2008, p. 72-73.

Jiji, 2009.
}

particular cargas de calefacción y/o refrigeración para una misma temperatura de confort), que los más extendidos y menores. Lo incidiría en el gasto requerido, y por ende, constituir uno de los aspectos esenciales del diseño sustentable. Sin embargo, esta vinculación no está cabalmente revisada en muchas situaciones, y la geometría de una construcción posee condiciones variadas, en especial respecto al desempeño térmico. Primeramente según la condición climática, ya que en las regiones cálidas este factor puede ser incluso inverso (una mayor superficie externa puede facilitar mayor ventilación natural y/o disipación del sobre-calentamiento). En las zonas frías, a su vez puede incidir la disposición respecto al asoleamiento. Además la edificación en sí misma, no posee una conformación continua, sino componentes variados y recintos independientes, que producen flujos internos y poseen distintas condiciones de transmitancia. Por la cual se requiere identificar la incidencia de la forma en tipologías constructivas y zonas climáticas específicas.

Por ejemplo, Depecker ${ }^{11}$ revisó catorce variaciones formales de un mismo edificio en distintas ciudades europeas, encontrando una relación de la compacidad con una menor demanda energética en los climas fríos, pero no así en los climas templados,

11 Depecker, Menezo, Virgone y Lepers, 2001. 
por lo que concluyó no recomendar consideraciones generales. Albaciti y Passerini ${ }^{12}$ lo comprobaron luego en edificaciones y regiones similares, encontrando una incidencia consistente pero más basada en la superficie asoleada. La norma europea EN $15217^{13}$ de desempeño energético de edificaciones menciona la relación entre superficie expuesta y volumen como un indicador, pero sin clarificar su incidencia en la demanda de las construcciones. La norma chilena Nch.1960 ${ }^{14}$ establece un procedimiento de cálculo de coeficientes volumétricos globales de pérdidas térmicas, que sugiere relaciones formales, pero no están expresadas, ni analizadas según condiciones constructivas específicas. El trabajo dirigido por Ourghi ${ }^{15}$ revisó edificios de oficinas advirtiendo una influencia combinada de la geometría, el área vidriada y tipo de vidrio, según distintos climas, comprobando una correlación directa entre la proporción de volumen y los requerimientos energéticos. Cappozoli ${ }^{16}$ lo comprobó también en varias ciudades italianas, aunque advirtió mayor incidencia de las ventanas. Tuhus-Dubrow y $\mathrm{Krarti}^{17}$ analizaron distintas formas de edificios residenciales mediante un sistema de simulación energética y optimización genética, identificando un mejor desempeño de las formas

12 Albaciti y Passerini, 2010.

13 CEN Comité Europeen de Normalisation, 2005.

14 INN Instituto Nacional de Normalización, 1989.

15 Ourghi, Al-Anzi y Krarti, 2007.

16 Capozzoli y Corrado, 2009.

17 Tuhus-Dubrow y Krarti, 2010. rectangulares y trapezoidales. Danielski ${ }^{18}$ estudió edificios residenciales colectivos de climas fríos, encontrando una reducción de $5 \mathrm{~kW}-\mathrm{h} / \mathrm{m}^{2}$ por cada 0.1 de la proporción general, pero advirtiendo la singularidad de las áreas comunes. También el trabajo dirigido por Parasonis ${ }^{19}$ analizó edificios residenciales multifamiliares en Lituania, verificando una incidencia hasta la mitad de la demanda energética por la compacidad del volumen, y sugiriendo un indicador general de área construida/superficie externa, con respecto a un edificio de referencia (lo que denominó "eficiencia geométrica relativa"). El estudio encabezado por $\mathrm{Choi}^{20}$ revisó varios edificios residenciales multifamiliares en Corea, encontrando un mayor consumo energético en las construcciones más altas que las bajas. Finalmente, en un reciente sumario de consideraciones energéticas para la edificación ${ }^{21}$, se sugiere un factor equivalente a la proporción de lados según la orientación, que incidiría en un 36\% de la calefacción en climas fríos.

Esta variedad de estudios expresa una influencia de condiciones formales en el comportamiento energético, en particular de la relación de superficie externa/volumen, pero con diversas singularidades,

\footnotetext{
Danielski, 2011.

Parasonis, Keizikas, Endriukaitytè y Kalibatienè, 2012.

Choia, Choa y Kim, 2012.

21 Pacheco, Ordoñez y Martínez, 2012.
} 
que amerita revisarlas en distintas situaciones. Además que cada tipología constructiva presenta variaciones geométricas particulares que podrían incidir de manera diferenciada, así como por orientación solar, acristalamiento o ventilación. Por otro lado, es relevante reconocer que la forma general de las edificaciones puede variar dentro de diversas consideraciones funcionales, urbanas o expresivas, con distribuciones y costos similares, lo que representa una potencialidad importante de mejoramiento energético.

\section{Características de las viviendas unifamiliares en el centro-sur de Chile}

La zona centro-sur de Chile (regiones del Maule, Bío-Bío y Araucanía) abarca una superficie aproximada de $100.000 \mathrm{~km}^{2}$ desde la latitud 34 4l' al norte, hasta los $39^{\circ} 37^{\prime}$ al sur, y entre la longitud $71^{\circ} 15^{\prime}$ hasta la $71^{\circ} 30^{\prime}$, de la costa del Pacífico hasta la Cordillera de los Andes. Posee un clima templado húmedo en toda la zona, con una radiación solar anual de $1.600 \mathrm{~kW}-\mathrm{h} / \mathrm{m}^{222}$, una media anual de temperatura cercana a los $13^{\circ} \mathrm{C}$, y una oscilación diaria de aprox. $12^{\circ} \mathrm{C}$ (mayor hacia el centro), según los antecedentes para la zona Sur-Litoral

22 CNE Comisión Nacional de Energía, 2008 de la norma chilena climática-habitacional ${ }^{23}$. Esta área alberga cerca de cuatro millones de habitantes en un millón de viviendas, que según el último censo publicado ${ }^{24}$, en un $94 \%$ son construcciones unifamiliares con propiedad individual del lote (casas), mayormente localizadas en zonas urbanas. Aunque esta cifra parece reducirse por recientes emprendimientos inmobiliarios en altura, expresa un amplio predominio residencial en extensión, similar a lo que acontece en la mayor parte del país y de muchas naciones en desarrollo. Con un proceso de edificación regular, de aproximadamente de 40.000 viviendas al año ${ }^{25}$, incrementadas recientemente por los procesos de reconstrucción del terremoto/tsunami de 2010 que afectó principalmente esta área. Aplicando desde el año 2000 y 2007 normativas térmicas con nuevas condiciones materiales de envolvente. Según los registros oficiales, estas edificaciones residenciales presentan una diversidad relevante entre construcciones aisladas o pareadas (cerca del 35 y 50\% respectivamente), con una baja participación de viviendas continuas (adosadas en dos o más costados). Del mismo modo en altura, poseen mayormente uno o dos pisos (aproximadamente en un 40\% de cada magnitud), lo que expresando una relevante variedad volumétrica individual. Ejecutadas con sistemas constructivos en madera y albañilería (en 49\%

\footnotetext{
23 INN Instituto Nacional de Normalización, 2008.

24 INE Instituto Nacional de Estadísticas, 2002.

25 MINVU Ministerio de Vivienda y Urbanismo, 2012.
} 
y $27 \%$ respectivamente), lo que suele estar combinado, porque la mayoría de las construcciones residenciales utiliza ambos materiales.Las magnitudes registradas de las viviendas revelan también una diversidad amplia desde $30 \mathrm{~m}^{2}$ hasta sobre $200 \mathrm{~m}^{2}$ de superficie por unidad, con una media cercana a los $60 \mathrm{~m}^{2}$, pero esto suele estar sub-representado por el amplio desarrollo de ampliaciones posteriores, que siguen condiciones constructivas similares a las iniciales, perono se registran.

Esta progresión de las edificaciones residenciales se advierte en todos los niveles sociales y sectores urbanos, produciendo una diferenciación volumétrica, y sustentando la preferencia por urbanizaciones horizontales que permiten un crecimiento individual. De manera distinta a otras naciones de bajos ingresos, que presentan también amplias magnitudes de edificación informal, pero más bien en áreas diferenciadas. Mientras en el país surge mayormente anexa a la construcción formal, y entretejida en la trama urbana. Respaldada por el desarrollo económico y la creciente disponibilidad de materiales y mano de obra, lo que sería característico de una nación emergente. Por esta razón, es más relevante caracterizar los aspectos formales de las construcciones residenciales, que se elaboran sucesivamente y de manera independiente.

\section{FIGURA 1. UBICACIÓN DEL ÁREA DE ESTUDIO Y LOCALIZACIÓN DE CASOS REGISTRADOS (AUTORES).}
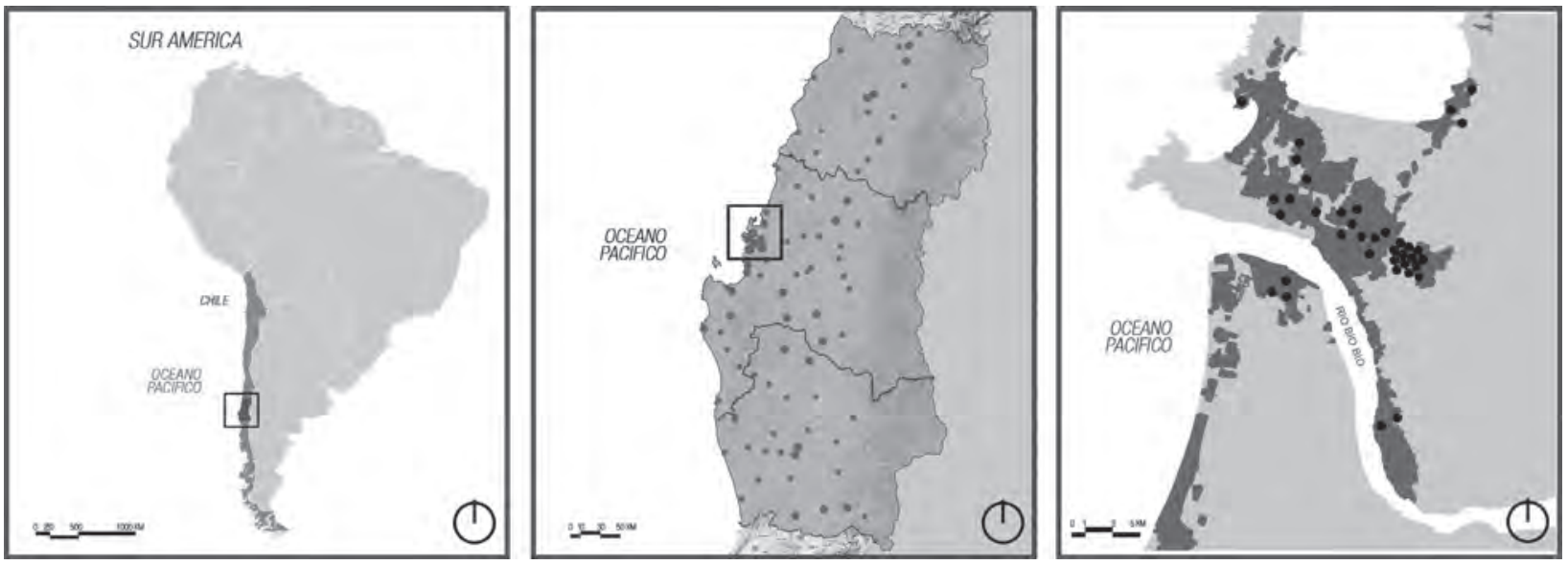

Fuente: Elaboración propia 


\section{FIGURA 2. VISTA DE ALGUNAS VIVIENDAS}

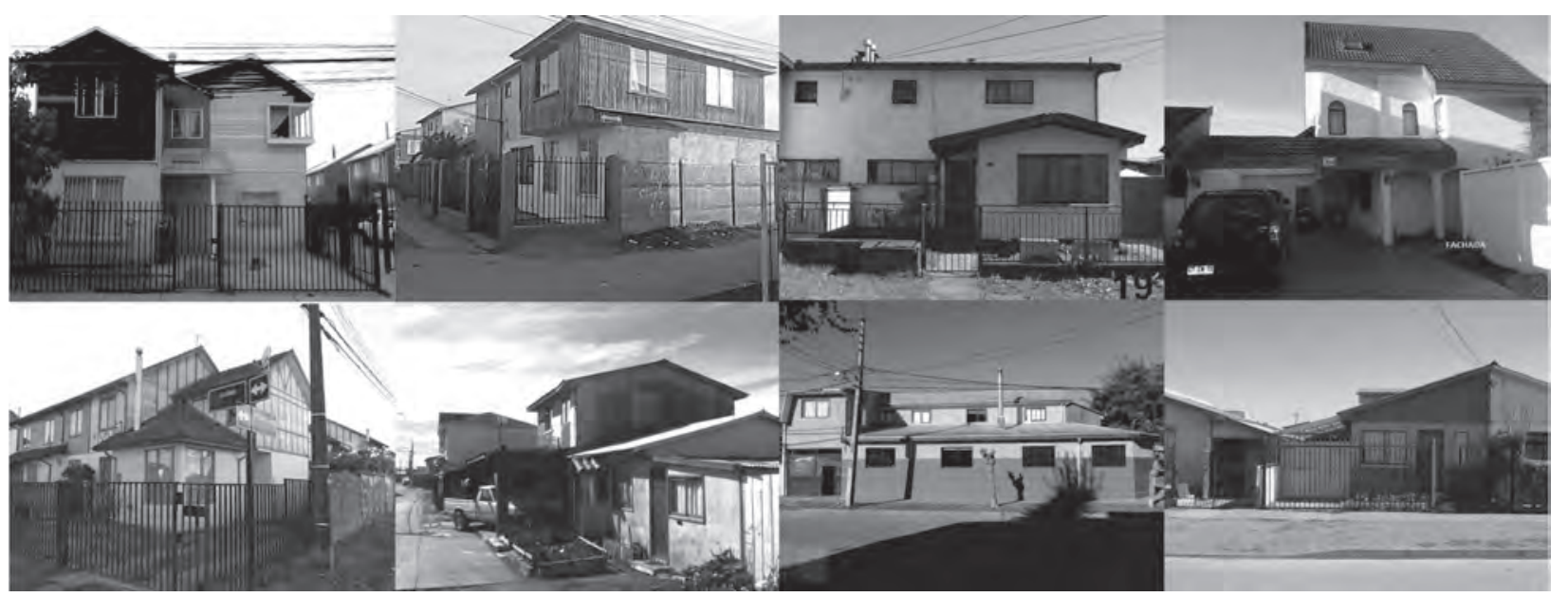

Fuente: Elaboración propia.

Para identificar más cabalmente las condiciones geométricas y ambientales de las viviendas en la zona,se revisaron en particular cincuenta viviendas del área metropolitana de Concepción (el principal conjunto urbano delazona). A través de la colaboración de estudiantes de arquitectura y construcción, quienes registraron sus propias residencias o de familiares próximos, con el fin de disponer de una muestra diversa con observadores capacitados. Se reguló la representatividad de los casos según las estadísticas globales, integrando en particular viviendas recientes y familias jóvenes (debido a que los hogares de los universitarios son usualmente más consolidados), y excluyendo residencias temporales, de modo que los estudiantes provenientes fuera del conjunto metropolitano compensaron la selección de casos con nuevas viviendas para ajustarlo a la variedad local. El origen socioeconómico de los estudiantes, adscritos a una universidad pública regional, refleja en general la 


\section{FIGURA 3. ESOUEMA DE FORMAS DE LAS VIVIENDAS REGISTRADAS}

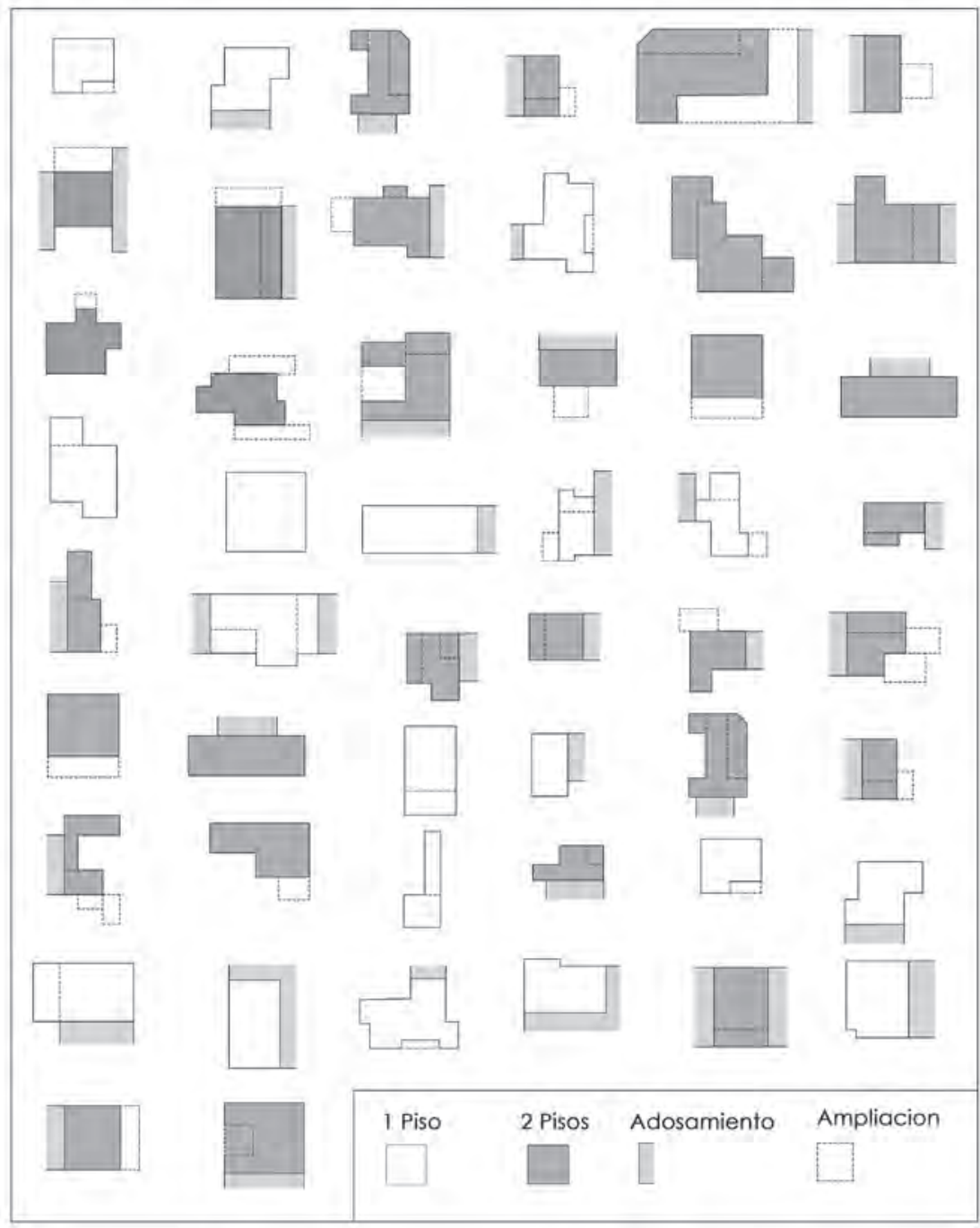

Fuente: Elaboración propia. 
diversidad de niveles de ingreso de la zona. Lo que se expresó en que las viviendas se localizaron en diversos sectores del área urbana (figura 1), con una antigüedad variada y regular (entre uno a sesenta años), y una representación de construcciones unifamiliares similar a los registros regionales y nacionales. Como se esperaba, la superficie de las viviendas presentó una magnitud mayor que las estadísticas existentes (con una media cercana a los $100 \mathrm{~m}^{2}$ ), ya que prácticamente todas poseían ampliaciones posteriores a la edificación original. Con una variedad de dimensiones entre 50 a 300 $\mathrm{m}^{2}$, dispersa en relación a los niveles socioeconómicos (más relacionada con la antigüedad de las viviendas).

Las viviendas unifamiliares revisadas presentaron en general una configuraciónortogonal centralizada (sin patios interiores), en uno o dos niveles de altura (figura 3). Ocasionalmente con diferencias en el segundo nivel y/o paramentos inclinados (ocupación de techumbres). Con una cantidad relevante de construcciones aisladas (41\%), algo mayor de adosadas en algunos de los costados (53\%), y unas pocas continúas (6\%), dispuestas en las distintas orientaciones cardinales. Aproximadamente la mitad presentó composiciones regulares, con cuatro paramentos, varias de longitudes casi equivalentes(cuadradas) o más extendidas en uno de los sentidos (rectangulares con proporciones desde 1,5 hasta 3). Las restantes poseen disposiciones irregulares con mayor cantidad de paramentos (seis, ocho o diez), en formas quebradas de L, T o Z. Las configuraciones materiales presentaron predominio de muros de albañilería con techumbres o segundos niveles de madera (65\%), seguidas por algunas construcciones mayormente de madera (29\%) y otras de hormigón armado. No se advirtieron diferencias sustanciales en los paramentos de las viviendas más recientes por las regulaciones térmicas aplicadas la última década, excepto en la aislación de techumbre,que también está presente en muchas de las construcciones anteriores.

Cabe mencionar que en estas viviendas se consultaron además patrones de ocupación, gasto energético y monitorización ambiental. Presentando una ocupación media regular de 4,5 habitantes (algo superiora las estadísticas oficiales) yperiodos extensos de utilización (sobre el 70\%), presumiblemente incididos por el asentamiento de las familias. Con servicios y equipamientos bastantes completos en todos los segmentos, asumiendo un gasto medio en electricidad de $2.100 \mathrm{~kW}$-h/año por vivienda al año, equivalentes aprox. a USD 600 anuales. Además de consumo de combustibles principalmente para calefacción, con un predominio de leña, además de gas y parafina, que totalizan desde

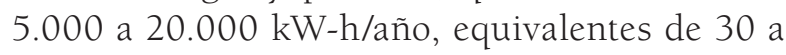
$130 \mathrm{~kW}$-h/año por m2. No se reconoce una diferencia relevante del gasto según la condición o antigüedad de las viviendas (o por la aplicación 
de las normas térmicas de la última década), las diferencias de montos corresponden mayormente al ingreso familiar. En los estudios nacionales y regionales disponiblesse reconoce un consumo medio similar por vivienda de $10.232 \mathrm{~kW}-\mathrm{h} /$ año ${ }^{26}$, y $13.500 \mathrm{~kW}-\mathrm{h} / \mathrm{año}^{27}$ respectivamente. Esto equivale a un gasto doméstico aproximado de USD 1.500 anuales, correspondientes a un 3 al 5\% del total de los ingresos familiares medios. Manteniendo un nivel de confort ambiental reducido (temperaturas medias entre 16 a $18^{\circ} \mathrm{C}$, con alta humedad y polución interior), especialmente en las viviendas de menores rentas con gastos bajos en calefacción, equipos menoresy más contaminantes, lo que también se ha verificado en estudios nacionales ${ }^{28}$. La reducción de los niveles globales de confort interior está influenciado también por la focalización horaria y espacial de los dispositivos de climatización (equipos móviles y temporales de calefacción), por la creciente individualización de la ocupación familiar y el escalamiento de los costos de combustible (que se expresa en el abandono de sistemas centralizados, y el incremento de fuentes locales más baratas pero contaminantes, como la leña). Revelando también la urgencia de adoptar medidas de mejoramiento ambiental residencial.

26 CDT Corporación de Desarrollo Tecnológico, 2010.

27 Fissore, 2012.

28 Sarmiento y Hormazabal, 2003.
Algunos estudios locales han revisado condiciones de desempeño ambiental de viviendas en la zona en relación a características formales. En un estudio de la consultora Ambientes ${ }^{29}$ se analizaron diez tipologías de viviendas representativas del país (de formas regulares, con distinta magnitud, altura y materialidad), en siete zonas climáticas con seis escenarios de mejoramiento material, efectuando 56 simulaciones en modo estático con grados-hora. Presentando para diez casos en Concepción demandas energéticas de 100 a $180 \mathrm{~kW}$-h/año por $\mathrm{m}^{2}$, fundamentalmente en calefacción, con incidencias relevantes por la altura, adosamiento vertical y horizontal, y materialidad (pero no de la superficie), aunque los casos no son equiparables. En el manual de vivienda social de Bustamante ${ }^{30}$ se exponen resultados de simulaciones dinámicas de viviendas similares realizadas con el software TAS, presentando para casos localizados en Concepción demandas de 60 a $140 \mathrm{~kW}$-h/año por $\mathrm{m}^{2}$, con relevantes reducciones por altura, y algo menores por proporción, disposición o contacto de las formas, y muy leves en orientación. Con una correlación general del factor de superficie externa/volumen, aunque con diversas alteraciones particulares. En el trabajo dirigido por Celis ${ }^{31}$ se analizaron con el software Casanova,diferentes viviendas en

\footnotetext{
29 Ambiente Consultores, 2007.

30 Bustamante, 2009, p. 73-74.

31 Celis, García, Trebilcock, Escorcia, Bruscato y Díaz, 2012.
} 
Concepción, con volúmenes regulares de distinta proporción, orientación y contigüidad, revelando demandas de 69 a $147 \mathrm{~kW}-\mathrm{h} / \mathrm{m}^{2}$ mayormente influenciadas por el adosamiento. La tesis doctoral de Hatt ${ }^{32}$ realizó con EnergyPlus y Genopt 20.736 simulaciones comparativas de viviendas con distintos parámetros, incluyendo un tercio localizadas en Concepción, con dos configuraciones formales y tres orientaciones que presentaron una incidencia global del 7\% y 2\% respectivamente. García y Broxford ${ }^{33}$ estudiaron con el software TAS ocho modelos regulares de viviendas en Santiago, en cuatro magnitudes, dos alturas, dos tipos de adosamiento y dos orientaciones, con tres escenarios de mejoramiento, revelando una fuerte incidencia de la superficie y la proporción, y algo más leve la baja altura, adosamiento y asoleamiento, además de una consistencia relevante en las medidas de mejoramiento.

Sin embargo, estos estudios no revisan específicamente la diversidad de variaciones formales en casos cabalmente comparables. De modo, que para revisar adecuadamente la incidencia de la variedad geométrica de la edificación residencial en zona, se requiere un análisis diferenciado y sustentado en las características específicas de las viviendas locales.

Hatt, 2012.

García y Broxford, 2012.

\section{Estudio de variaciones formales y simulaciones energéticas}

Con el fin de comprobar las incidencias formales en el desempeño energético de viviendas del centro-sur de Chile, se estudiaron 128 variaciones de una construcción similar, mediante simulaciones energéticas realizadas con el software Ecotect para modelación, y EnergyPlus como motor de cálculo. Considerando cinco parámetros combinados; altura, proporción, extensión, contigüidad, orientación, que presentan alteraciones frecuentes en las construcciones habitacionales en la zona según la revisión efectuada, y eventuales influencias relevantes de acuerdo aestudios anteriores. En ciertas magnitudes significativas por cada parámetro, basadas en las condiciones usuales de edificación local registradas en las estadísticas y en las viviendas estudiadas. Analizadas de manera integrada en una situación residencial equivalente, es decir con una misma superficie construida, especificación material y localización, así como similares características arquitectónicas y de ocupación. De modo que fueran equiparables exclusivamente por las diferencias geométricas, 
TABLA 1. ESPECIFICACIÓN CONSTRUCTIVA Y VALORES DE TRANSMITANCIA DE LA VIVIENDA SIMULADA.

\begin{tabular}{|c|c|c|c|c|c|}
\hline Elemento & $\begin{array}{l}\text { Materialidadespesor } \\
\mathrm{mm}\end{array}$ & $\begin{array}{l}\text { Densidad } \\
\text { aparente } \\
\mathrm{kg} / \mathrm{m}^{3}\end{array}$ & $\begin{array}{l}\text { Conductividad } \\
\text { térmica, }, \\
\mathrm{W} /(\mathrm{m} \times \mathrm{K})\end{array}$ & Calor especifico & $\begin{array}{l}\text { valor U W/(m2 } \\
\mathrm{x} \mathrm{K)}\end{array}$ \\
\hline \multirow[t]{2}{*}{ MUROS } & ESTUCO (2) & 2.000 & 1,4 & 1.088 & \multirow[t]{2}{*}{2,39} \\
\hline & LADRILLO(140) & 1.400 & 0,6 & 840 & \\
\hline PISO & RADIER(100) & 2.400 & 1,63 & 920 & 4,32 \\
\hline \multirow{3}{*}{ ENTREPISO } & YESO CARTON (10) & 700 & 0,26 & 840 & \multirow[t]{3}{*}{1,91} \\
\hline & MADERA (18) & 410 & 0,104 & 1.088 & \\
\hline & ALFOMBRA (15) & 1.000 & 0,05 & 732,2 & \\
\hline \multirow[t]{2}{*}{ CIELO } & YESO CARTON (10) & 700 & 0,26 & 840 & \multirow[t]{2}{*}{0,48} \\
\hline & POLIESTIRENO (80) & 20 & 0,043 & 1.700 & \\
\hline TECHO & PLANCHA DE ZINC $(0,4)$ & 700 & 58 & 390 & 5,88 \\
\hline
\end{tabular}

Fuente: Elaboración propia.

y esclarecer de este modo la participación de las condiciones formales en el comportamiento ambiental.

Para todas las variaciones se estableció una misma superficie de $72 \mathrm{~m}^{2}$ de edificación, que corresponde a una media aproximada de las viviendas unifamiliares en la zona, que permitiría diferentes distribuciones de planta. Con muros de ladrillo estucados con cemento $(\mathrm{U}=2.39)$, suelo de hormigón en contacto con el terreno ( $\mathrm{U}=4.32)$, cielos de yeso-cartón, con aislante de poliestireno $(\mathrm{U}=0.48)$, con techumbres inclinadas sin ventilar y cubiertas con planchas de zinc $(U=5.88)$, entrepisos y divisiones interiores de estructura de madera revestidas con yeso-cartón $(U=1.91)$, según especificación y condiciones materiales requeridos por el software para la simulación (tabla 1), indicando valores de transmitancia de cada elemento como 
referencia ${ }^{34}$. Estas condiciones son levemente inferiores a la normativa vigente desde el año 20002007 para esta zona ${ }^{35}$, pero más aproximadas a la edificación residencial existente y la efectivamente realizada, ya que usualmente no se aplican cabalmente y tampoco se fiscalizan en terreno. Considerando además que las características térmicas de los muros, e incluso lo requerido por las normas para la zona $(\mathrm{U}=1,7)$ es particularmente débil frente a los estándares internacionales, lo que afecta las configuraciones. Se contempló un porcentaje regular de vanos del $30 \%$ de la superficie vertical expuesta, que es una proporción usual en las construcciones residenciales en la zona, aún en distintas disposiciones. Con ventanas de tamaño mediano, centradas ydistribuidas igualmente en cada una de las caras (es decir,similarespor costado), con marcos metálicos de 2,5 mm. y vidrios simples (se excluyeron puertas exteriores por su incidencia leve). Con una altura interior de los pisos de $2.40 \mathrm{~m}$, y divisiones de recintos aproximadamente cada $3 \mathrm{~m}$.

34 Se debe considerar que según Nch853 (INN, 2007) el cálculo de transmitancia de suelos depende del diferencial de temperatura de terreno, el entrepiso no se contempla para análisis de envolvente, $y$ en el complejo de techumbre se integra cielo y cubierta con la cámara de aire, pero en este estudio por simulación, el cálculo se realiza en el software según la base climática y análisis dinámico.

35 Reglamentación Térmica; Art. 4.1.10 de la Ordenanza General de Urbanización y Construcciones (OGUC)
Las alteraciones o parámetros formales estudiados son;

- Altura: en uno o dos pisos equivalentes (no se contemplaron variaciones entre pisos o segundos pisos con muros inclinados por su excesiva diferenciación, y su incidencia parece menor);

- Proporción; fundamentalmente entre una configuración regular entre una relación 1:1 equivalente entre los lados (base cuadrada), o de 1:2 (base rectangular)

- Extensión; considerada como la cantidad de caras verticales en planta, con las cuadradas y rectangulares de cuatro caras, una disposición quebrada (L) con seis, y otra de doble quiebre (Z) con ocho. Expresando de este modo la diversidad de configuraciones que se pueden presentar (como disposiciones en $\mathrm{T}, \mathrm{Cu}$ otros quiebres), fundamentalmente por la cantidad de aristas (que producen puentes térmicos y cambios de captación solar), lo que constituye un aspecto relevante de la variedad formal. 


\section{FIGURA 4. MODELOS BÁSICOS DE LAS VIVIENDAS SIMULADAS EN AXONOMETRICAS 80\%10}

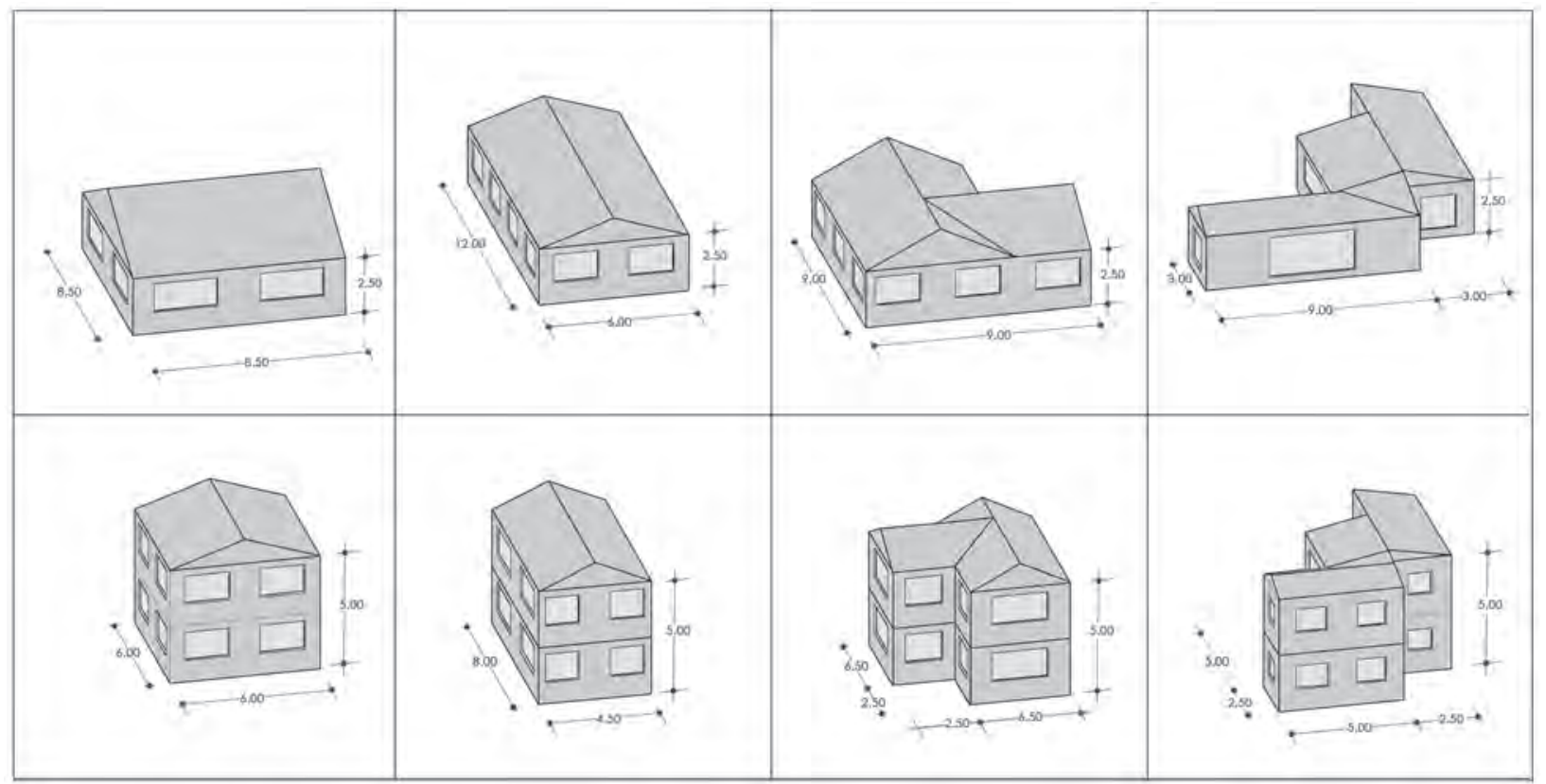

Fuente: Elaboración propia.

- Contigüidad, relación con volúmenes vecinos en condición aislada, adosamiento por el lado mayor o menor o en ambos (continuo).

- Orientación, en los cuatro sentidos principales (norte, este, sur, oeste).

De los cuales dos (proporción y extensión), corresponden a variaciones de morfología que se contemplan conjuntamente en cuatro alternativas; cuadrada, rectangular, quebrada y doble quebrada. Las tres primeras definen configuraciones generales de los volúmenes, por lo que combinadas generan ocho modelos básicos $(2 * 2 * 2)$, sobre los cuales se desarrollan las cuatro condiciones de contigüidad, es decir treinta y dos condiciones distintas, estudiadas en cuatro orientaciones diferentes, definiendo entonces 128 casos. Como 


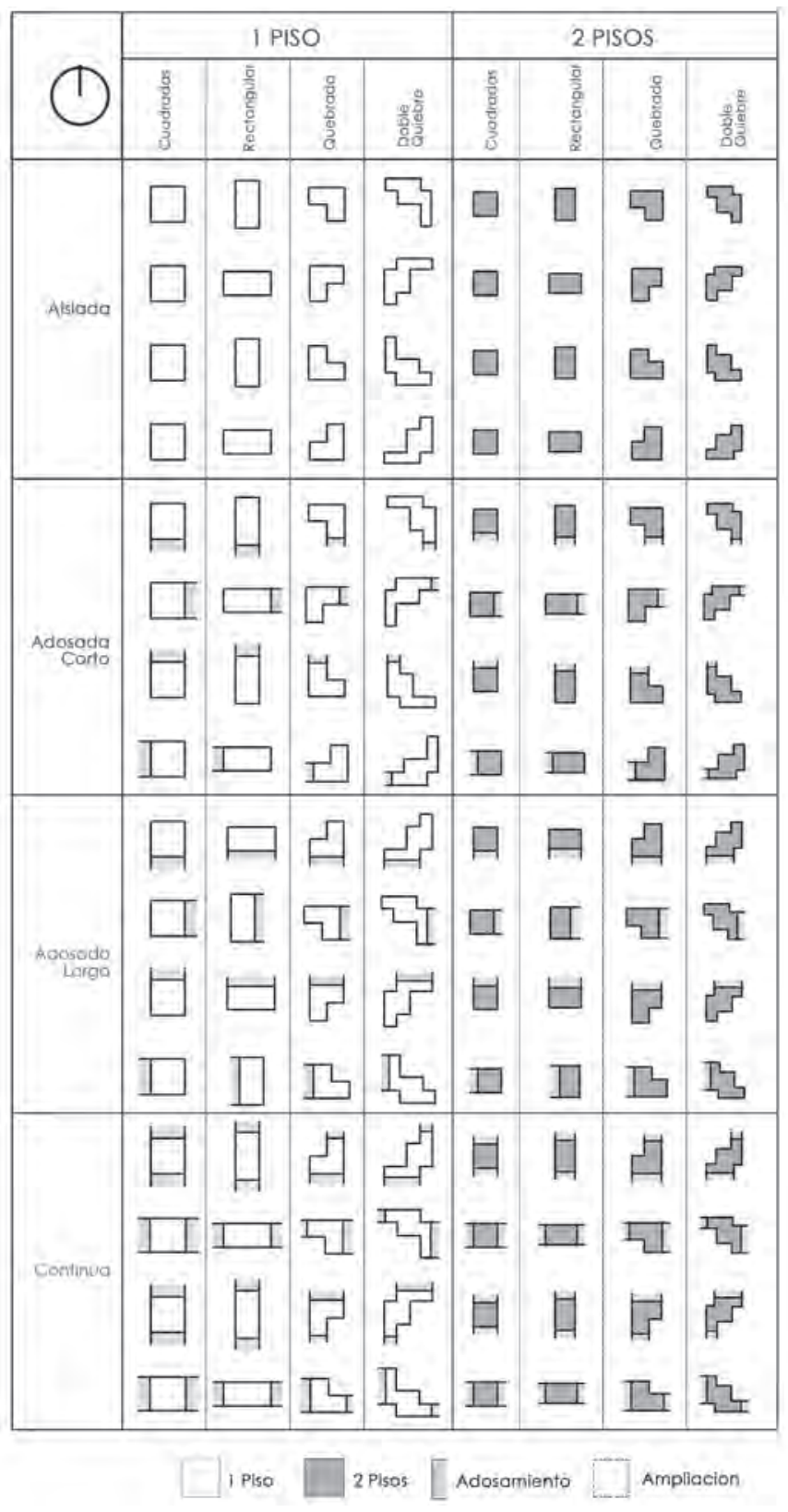

\section{FIGURA 5. VARIACIONES DE LAS VIVIENDAS} ESTUDIADAS

Fuente: Elaboración propia. 


\section{FIGURA 6. SIMULACIÓN ENERGÉTICA DE LOS MODELOS EN ECOTECT+ENERGYPLUS.}

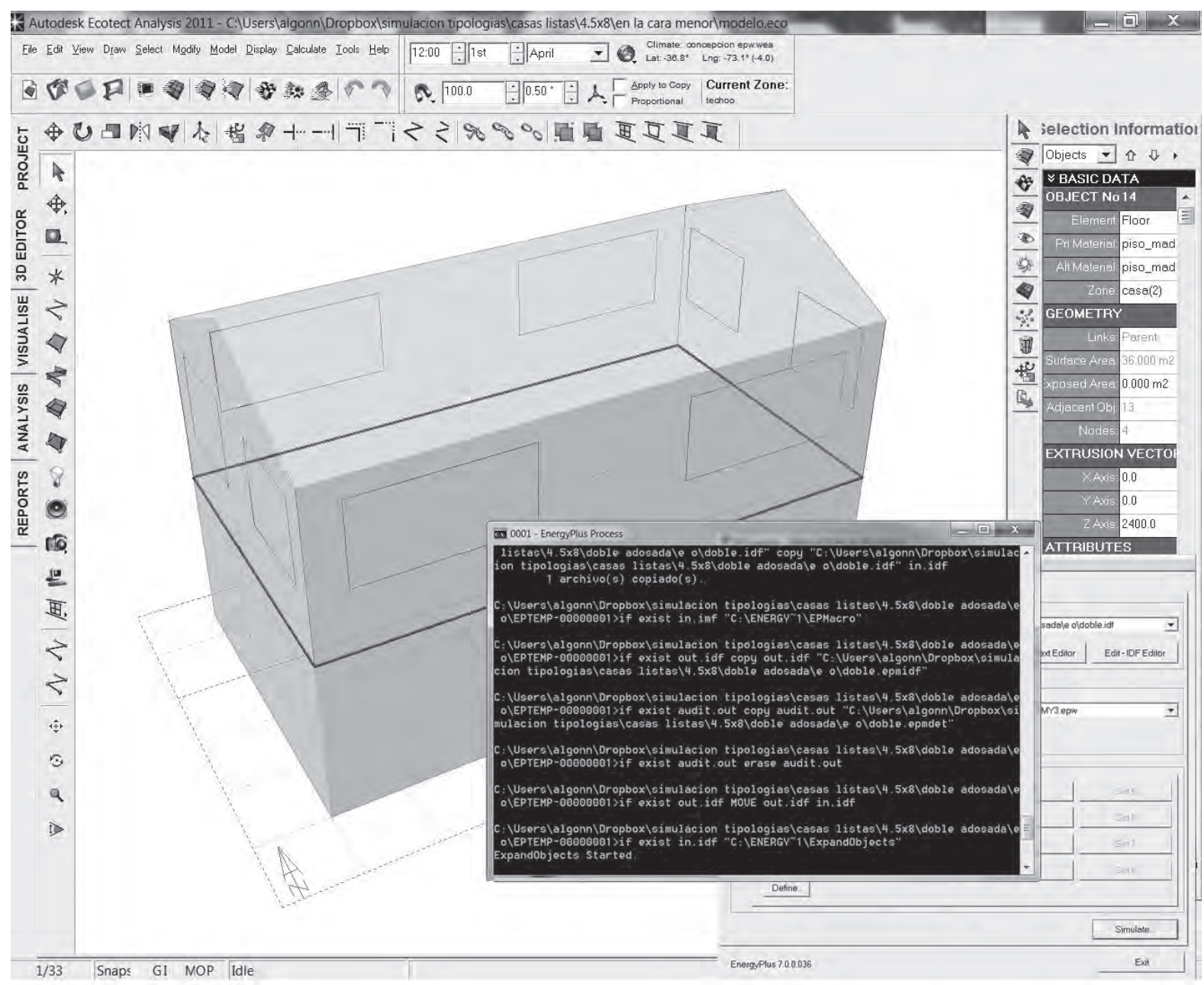

Fuente: Elaboración propia. 


\section{FIGURA 7. EJEMPLO DE VALORES MENSUALES DE DEMANDA ENERGÉTICA POR VIVIENDA.}

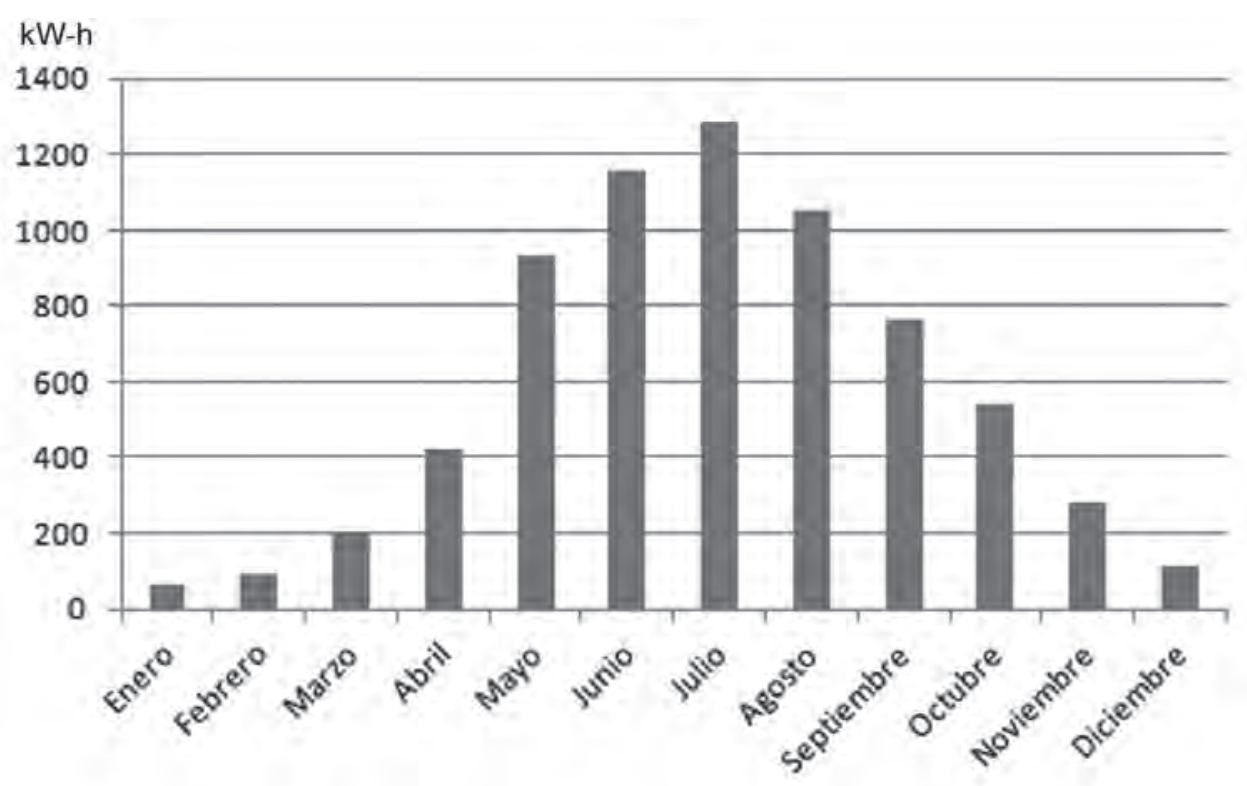

Fuente: Elaboración propia.

varias de éstas son simétricas (por ejemplo las regulares aisladas y adosamientos respecto a las distintas orientaciones), son setenta y dos modelos simulados, pero igualmente se contabilizan los resultados de las todas las variaciones para revisar la incidencia consolidada de cada parámetro.
Las simulaciones energéticas consideraron una banda de confort de $18^{\circ} \mathrm{C}$ a $24^{\circ} \mathrm{C}$, según calculo adaptativo para la zona, y base climática IWEC ${ }^{36}$ para la ciudad de Concepción, que presenta una mayor fidelidad a los registros meteorológicos, que la base normalmente utilizada en

36 ASHRAE, s.f. 
TABLA 2: DEMANDA ANUALMEDIA POR SUPERFICIE EN CADA VARIACIÓN, SEGÚN FIGURA 4.

\begin{tabular}{|c|c|c|c|c|c|c|c|c|c|c|}
\hline \multirow{2}{*}{\multicolumn{2}{|c|}{$\begin{array}{l}\text { DEMANDA (KW-H/ } \\
\text { M2) }\end{array}$}} & \multicolumn{4}{|l|}{1 PISO } & \multicolumn{4}{|l|}{2 PISOS } & \multirow[t]{2}{*}{ Promed. } \\
\hline & & CUADRADA & $\begin{array}{l}\text { RECTAN- } \\
\text { GULAR }\end{array}$ & QUEBRADA & $\begin{array}{l}\text { DOBLE } \\
\text { QUEBRADA }\end{array}$ & CUADRADA & $\begin{array}{l}\text { RECTAN- } \\
\text { GULAR }\end{array}$ & QUEBRADA & $\begin{array}{l}\text { DOBLE } \\
\text { QUEBRADA }\end{array}$ & \\
\hline \multirow[t]{4}{*}{ AISLADA } & NORTE & 114,97 & 121,42 & 128,74 & 165,75 & 168,32 & 182,15 & 192,97 & 231,76 & \multirow{4}{*}{163.35} \\
\hline & ESTE & 114,97 & 121,04 & 129,28 & 166,20 & 168,32 & 180,47 & 193,25 & 232,09 & \\
\hline & SUR & 114,97 & 121,42 & 129,53 & 166,17 & 168,32 & 182,15 & 193,35 & 232,29 & \\
\hline & OESTE & 114,97 & 121,04 & 129,28 & 167,68 & 168,32 & 180,47 & 193,35 & 232,18 & \\
\hline \multirow{4}{*}{$\begin{array}{l}\text { ADOSADA } \\
\text { CORTO }\end{array}$} & NORTE & 108,81 & 118,59 & 118,64 & 151,87 & 152,09 & 166,60 & 174,88 & 219,57 & \multirow{4}{*}{147,84} \\
\hline & ESTE & 101,81 & 115,15 & 114,44 & 152,21 & 148,33 & 165,02 & 173,32 & 219,89 & \\
\hline & SUR & 93,71 & 108,81 & 108,71 & 152,07 & 146,82 & 158,02 & 168,22 & 215,61 & \\
\hline & OESTE & 101,37 & 114,60 & 113,88 & 153,17 & 140,63 & 163,79 & 172,06 & 218,24 & \\
\hline \multirow{4}{*}{$\begin{array}{l}\text { ADOSADA } \\
\text { LARG0 }\end{array}$} & NORTE & 108,81 & 110,35 & 114,13 & 143,64 & 152,09 & 114,59 & 163,36 & 206,09 & \multirow{4}{*}{133,80} \\
\hline & ESTE & 101,81 & 99,28 & 107,33 & 140,30 & 148,33 & 115,15 & 159,56 & 205,77 & \\
\hline & SUR & 93,71 & 88,31 & 99,32 & 134,03 & 146,82 & 108,81 & 151,77 & 204,10 & \\
\hline & OESTE & 101,37 & 98,47 & 106,65 & 139,82 & 140,63 & 114,60 & 157,75 & 204,95 & \\
\hline \multirow[t]{4}{*}{ CONTINUA } & NORTE & 80,10 & 99,33 & 106,73 & 137,98 & 108,60 & 92,50 & 135,98 & 192,60 & \multirow{4}{*}{118,58} \\
\hline & ESTE & 80,84 & 98,88 & 103,10 & 138,66 & 111,26 & 90,90 & 135,41 & 191,66 & \\
\hline & SUR & 80,10 & 99,33 & 99,33 & 137,98 & 108,60 & 92,50 & 130,19 & 192,60 & \\
\hline & OESTE & 80,84 & 98,88 & 102,76 & 138,66 & 111,26 & 90,90 & 134,51 & 191,66 & \\
\hline \multirow[t]{2}{*}{ promedios } & & 99,57 & 108,43 & 116,66 & 152,39 & 143,05 & 137,41 & 174,49 & 212,11 & \multirow[t]{2}{*}{143,01} \\
\hline & & 119,26 & & & & 166,76 & & & & \\
\hline
\end{tabular}

Fuente: Elaboración propia.

las simulaciones energéticas (Meteonorm). No se consideró ocupación ni equipamientos de climatización en las simulaciones para descontar las incidencias temporales. Obteniendo un gráfico de demandas energéticas mensuales que reflejan la variación estacional del clima local, concentrándose mayormente en calefacción para el periodo de invierno entre mayo a octubre.
Persistiendo leves demandas de calefacción en todos los restantes meses (usualmente para noches y mañana frías), y muy escasas magnitudes de refrigeración en algunos meses de verano (enero-febrero). Las demandas anuales fueron divididas por la superficie construida para utilizarla como indicador de comparación y comprobarlo con otros estudios similares. 


\section{Análisis de resultados de demanda energética}

Las simulaciones realizadas generaron demandas anuales desde $80,1 \mathrm{~kW}-\mathrm{h} / \mathrm{m}^{2}$ (en el modelo cuadrado de un piso con doble adosamiento nortesur) hasta $231,76 \mathrm{~kW}-\mathrm{h} / \mathrm{m}^{2}$ (en el modelo doble quebrado aislado de dos pisos), con una distribución variada entre ambos, lo que revela una incidencia relevante de aspectos formales en el desempeño, aunque dispersa. Con una media global de $142,94 \mathrm{~kW}-\mathrm{h} / \mathrm{m}^{2}$, lo que es cercano a otros estudios en la zona ${ }^{37}$.

La característica que presentó una mayor diferencia global fue la extensión(variación morfológica). Los casos de doble quiebre en ambas alturas presentaron una demanda media de $182,25 \mathrm{~kW}-\mathrm{h} / \mathrm{m}^{2}$, casi el 50\% superior a los de forma cuadrada, con una media entre ambas de $121,31 \mathrm{~kW}-\mathrm{h} / \mathrm{m}^{2}$, con una magnitud progresiva de valores intermedios consecutivos, de los rectangulares y quebrados. Esto implica que, con una configuración constructiva similar en la zona, un perfil más prolongado y variable puede provocar cerca de la mitad del gasto energético y/o deteriorar sensiblemente la calidad ambiental de una vivienda, independiente del

37 Ambientes 2007; Bustamante, 2009; Celis, García, Trebilcock, Escorcia, Bruscato, y Díaz, 2012; Hatt, 2012. detalle u orientación, y en una magnitud mayor que las incidencias materiales ${ }^{38}$. Probablemente por el aumento de caras exteriores, la obstrucción solar de los muros, mayores puentes térmicos de las aristas, etc. Esta condición ha sidoadvertida en las referencias generales del tema, pero escasamente revisada en los estudios locales ${ }^{39}$, quizás por las dificultades de modelación que implican. Cabe mencionar también que en las viviendas existentes analizadas de distintos sectores o periodos, más de la mitad, presentaban trazados irregulares. Además este aspecto no suele estar definido por requerimientos específicos, sino motivado fundamentalmente por una variedad formal (que igualmente se puede lograr con tratamientos menores). Ciertamente una disposición irregular permite distribuciones interiores más distintas y ocasionalmente mejor iluminación o ventilación natural en los recintos (con ventanas en distintos costados), pero también mayores costos de ejecución y mantención (por el aumento de muros, techumbres y encuentros). De modo que si la regularidad volumétrica contribuye sustancialmente a la reducción energética, podría promoverse sin mayores conflictos en la edificación.

La proporción, que constituye la distinción entre los casos cuadrados y rectangulares, presentó una

38 Según estudios previos que indican variaciones hasta el $30 \%$ (Bustamante, 2009).

39 Solo considerada parcialmente en Bustamante, 2009 y Hatt, 2012. 
incidencia global casi nula, debido a que variaban inversamente y en magnitudes leves por los adosamientos y alturas. Las formas rectangulares presentaron una demanda media un $7 \%$ superior en condiciones aisladas, especialmente en dos pisos, pero un 7\% inferior en adosamientos mayores. Los documentos generales de arquitectura bio-climática sugieren usualmente una relación diferenciada de lados en los volúmenes (formas rectangulares), especialmente vinculada a la orientación solar y vientos locales. Varios análisis en la zona comparan casos de distintas proporciones, expresando una demanda levemente inferior en los más cuadrados, pero también con discrepancias ${ }^{40}$. Por lo que podemos afirmar que esta condición no es muy determinante para las viviendas en la región, o al menos que no son concluyentes los estudios al respecto.

La relación de contigüidad entre las viviendas es otro aspecto que presenta diferencias relevantes en los resultados de demanda energética. Las viviendas aisladas en total presentaron una media de $163,35 \mathrm{~kW}-\mathrm{h} / \mathrm{m}^{2}$ que es un $38 \%$ más alta que la media de las continuas con 118,58 kW-h/m², con los adosamientos en valores intermedios. Lo que expresa una reducción relevante por la contigüidad, que es reconocido también en varios trabajos

40 Bustamante, p. 114, se invierte esta situación con dos pisos. con una divergencia similar ${ }^{41}$. Lo que evidencia que una vivienda en la zona por el solo hecho de distanciarse del vecino incrementa en un tercio su demanda energética, o en su defecto reduce su confort interior (lo que sucede en sectores de bajos ingresos que no pueden destinar fondos adecuados para climatización). Pero se debe reconocer que la independencia volumétrica está relacionada con otros aspectos de habitabilidad (aislamiento acústico, mayor luminosidad y ventilación natural, retardo de propagación del fuego), como también de identidad social. Pero a la vez de mayor mantención (por mas fachadas expuestas), y extensión urbana (por requerir sitios mayores), que redunda a su vez en incrementar las distancias de transporte (y por ende el tiempo, contaminación y combustibles dedicado a esta actividad), debilitando el acceso a equipamientos y promoviendo segregación social, como ha demostrado esta tipología residencial en los países subdesarrollados. Las relevantes reducciones energéticas expresadas por la contigüidad residencial y su contribución a la densidad y sostenibilidad urbana, deberían por tanto fomentar esta tipología, respaldadas por un mejoramiento de las condiciones técnicas de habitabilidad y de expresión arquitectónica para su mejor calidad residencial y significación social.

41 Ambientes, 2007; Bustamante, 2009; Celis, García, Trebilcock, Escorcia, Bruscato, y Díaz, 2012. 
La altura de las viviendas también reveló una variación importante en las demandas, presentando una media de $166,76 \mathrm{~kW}-\mathrm{h} / \mathrm{m}^{2}$ en todas las de dos pisos y $119,26 \mathrm{~kW}-\mathrm{h} / \mathrm{m}^{2}$ en todas las de un piso, incrementándose especialmente en las irregulares. Esta reducción en las bajas alturas parece contradictoria con sugerencias generales que promueven construcciones bajas (motivadas por rebajar la superficie global expuesta), como también en un par de revisiones locales ${ }^{42}$, aunque de manera irregular. Sin embargo, es consistente en todos los modelos estudiados y con la concentración de demanda en calefacción, ya que las bajas temperaturas promueven mayor relevancia de las masas térmicas y de la baja transmitancia de los muros, que en estas viviendas de menor altura se reduce por la mayor superficie de suelo y cielo (que posee una aislación adecuada), además de menor magnitud absoluta de vanos y paramentos. Aunque también las edificaciones residenciales en baja altura reducen la calidad ambiental de sus recintos (por menor iluminación y ventilación) y requieren mayores lotes, por tanto promueven la extensión residencial, con los deterioros consecuentes de sostenibilidad urbana. De modo que parece una característica apropiada para promover en casos singulares, pero de menor relevancia ambiental en términos generales.

42 Ambientes, 2007 y Bustamante, 2009.
La orientación fue de escasa significancia en los distintos casos estudiados. Todos los modelos con mayor disposición solar (fachadas más abiertas y extensas al norte) presentaron una demanda media de 137,94 kW-h/m², mientras las inversas obtuvieron una media de $143,45 \mathrm{~kW}-\mathrm{h} / \mathrm{m}^{2}$, o sea apenas un $4 \%$ mejor (aunque consistente). Esto también contrasta con las reiteradas afirmaciones de los documentos de arquitectura bio-climática sobre la relevancia de la orientación solar, especialmente para sistemas de captación pasiva. Sin embargo, aparentemente en estas zonas templadas de baja radiación y edificaciones de aperturas regulares no tiene mayor incidencia en las demandas energéticas y los estudios de viviendas en la zona concuerdan en este sentido ${ }^{43}$. Pero ciertamente es recomendable, en particular para acciones de ganancias térmicas.

\section{Factor de regulación}

Estos resultados permiten advertir la incidencia de diversos aspectos formales en los requerimientos energéticos habitacionales en el centro-sur de Chile, de manera consistente, pero diferenciada. Con el fin de identificar un indicador que, para esta tipología y zona climática expresara las variaciones energéticas según la forma, se aplicaron diferentes mediciones geométricas a los modelos. Revisando magnitudes

43 Bustamante, 2009; Celis, García, Trebilcock, Escorcia, Bruscato, y Díaz, 2012; Hatt, 2012. 


\section{TABLA 3: DEMANDA ENERGÉTICA (EN KW-H/M2) SEGÚN VALORES GEOMÉTRICOS (MEDICIONES Y RELACIONES) DE LAS ALTERNATIVAS MORFOLÓGICAS SEGÚN ALTURAS Y ADOSAMIENTOS.}

\begin{tabular}{|c|c|c|c|c|c|c|c|c|c|c|c|c|c|c|c|c|}
\hline \multirow[t]{4}{*}{ CASOS } & \multicolumn{16}{|c|}{$1 \mathrm{PISO}$} \\
\hline & \multicolumn{4}{|c|}{ CUADRADA } & \multicolumn{4}{|c|}{ RECTANGULAR } & \multicolumn{4}{|c|}{ QUEBRADA } & \multicolumn{4}{|c|}{ DOBLE QUEBRADA } \\
\hline & AISL & $\begin{array}{l}\mathrm{AD} \\
\mathrm{CT}\end{array}$ & $\begin{array}{l}\text { AD } \\
\text { LR }\end{array}$ & CONT & AISL & $\begin{array}{l}\mathrm{AD} \\
\mathrm{CT}\end{array}$ & $\begin{array}{l}\text { AD } \\
\text { LR }\end{array}$ & CONT & AISL & $\begin{array}{l}\mathrm{AD} \\
\mathrm{CT}\end{array}$ & $\begin{array}{l}\text { AD } \\
\text { LR }\end{array}$ & CONT & AISL & $\begin{array}{l}\mathrm{AD} \\
\mathrm{CT}\end{array}$ & $\begin{array}{l}\text { AD } \\
\text { LR }\end{array}$ & CONT \\
\hline & $1 \mathrm{CA}$ & $1 \mathrm{CC}$ & $1 \mathrm{CL}$ & $1 \mathrm{CN}$ & 1RA & $1 \mathrm{RC}$ & $1 \mathrm{RL}$ & 1RN & $10 \mathrm{~A}$ & $10 \mathrm{C}$ & $10 \mathrm{~L}$ & $10 \mathrm{~N}$ & 1DA & $1 \mathrm{DC}$ & $1 \mathrm{DL}$ & 1DN \\
\hline $\begin{array}{l}\text { DEMANDA } \\
\mathrm{kW}-\mathrm{h} / \mathrm{m} 2\end{array}$ & 115 & 101,4 & 101,4 & 80,47 & 121,1 & 114,3 & 99,1 & 99,1 & 128,7 & 113,9 & 106,9 & 103 & 165,8 & 152 & 145,2 & 138,3 \\
\hline LARGO (ml) & 8,5 & 8,5 & 8,5 & 8,5 & 12 & 12 & 12 & 12 & 9 & 9 & 9 & 9 & 12 & 12 & 12 & 12 \\
\hline ANCHO (ml) & 8,5 & 8,5 & 8,5 & 8,5 & 6 & 6 & 6 & 6 & 6 & 6 & 6 & 6 & 12 & 12 & 12 & 12 \\
\hline LADOS (cant.) & 4 & 3 & 3 & 2 & 4 & 3 & 3 & 2 & 6 & 5 & 5 & 4 & 10 & 9 & 9 & 8 \\
\hline PERIMETRO (ml) & 34 & 25,5 & 25,5 & 17 & 36 & 30 & 24 & 24 & 36 & 30 & 27 & 21 & 48 & 45 & 39 & 36 \\
\hline ALTO (ml) & 2,4 & 2,4 & 2,4 & 2,4 & 2,4 & 2,4 & 2,4 & 2,4 & 2,4 & 2,4 & 2,4 & 2,4 & 2,4 & 2,4 & 2,4 & 2,4 \\
\hline MUROS $\left(\mathrm{m}^{2}\right)$ & 81,6 & 61,2 & 61,2 & 40,8 & 86,4 & 72 & 57,6 & 57,6 & 86,4 & 72 & 64,8 & 50,4 & 115,2 & 108 & 98,6 & 86,4 \\
\hline CIELOS $\left(\mathrm{m}^{2}\right)$ & 72 & 72 & 72 & 72 & 72 & 72 & 72 & 72 & 72 & 72 & 72 & 72 & 72 & 72 & 72 & 72 \\
\hline SUP. EXP. $\left(\mathrm{m}^{2}\right)$ & 153,6 & 133,2 & 133,2 & 112,8 & 158,4 & 144 & 129,6 & 129,6 & 158,4 & 144 & 136,8 & 122,4 & 187,2 & 180 & 165,6 & 158,4 \\
\hline PER/AREA & 0,47 & 0,35 & 0,35 & 0,24 & 0,50 & 0,42 & 0,33 & 0,33 & 0,50 & 0,42 & 0,38 & 0,29 & 0,67 & 0,63 & 0,54 & 0,50 \\
\hline MUR/AREA & 1,13 & 0,85 & 0,85 & 0,57 & 1,20 & 1,00 & 0,80 & 0,80 & 1,20 & 1,00 & 0,90 & 0,70 & 1,60 & 1,50 & 1,30 & 1,20 \\
\hline SUP/AREA & 2,13 & 1,85 & 1,85 & 1,57 & 2,20 & 2,00 & 1,80 & 1,80 & 2,20 & 2,00 & 1,90 & 1,70 & 2,60 & 2,50 & 2,30 & 2,20 \\
\hline PER(VOL & 0,20 & 0,15 & 0,15 & 0,10 & 0,21 & 0,17 & 0,14 & 0,14 & 0,21 & 0,17 & 0,16 & 0,12 & 0,28 & 0,26 & 0,23 & 0,21 \\
\hline MUR/VOL & 0,47 & 0,35 & 0,35 & 0,24 & 0,50 & 0,42 & 0,33 & 0,33 & 0,50 & 0,42 & 0,38 & 0,29 & 0,67 & 0,63 & 0,54 & 0,50 \\
\hline SUP/VOL (FF) & 0,89 & 0,77 & 0,77 & 0,65 & 0,92 & 0,83 & 0,75 & 0,75 & 0,92 & 0,83 & 0,79 & 0,71 & 1,08 & 1,04 & 0,96 & 0,92 \\
\hline
\end{tabular}

Fuente: Elaboración propia. 


\section{FIGURA 8. GRÁFICO DE MEDICIONES GEOMÉTRICAS SEGÚN CASOS ORDENADOS POR DEMANDA.}

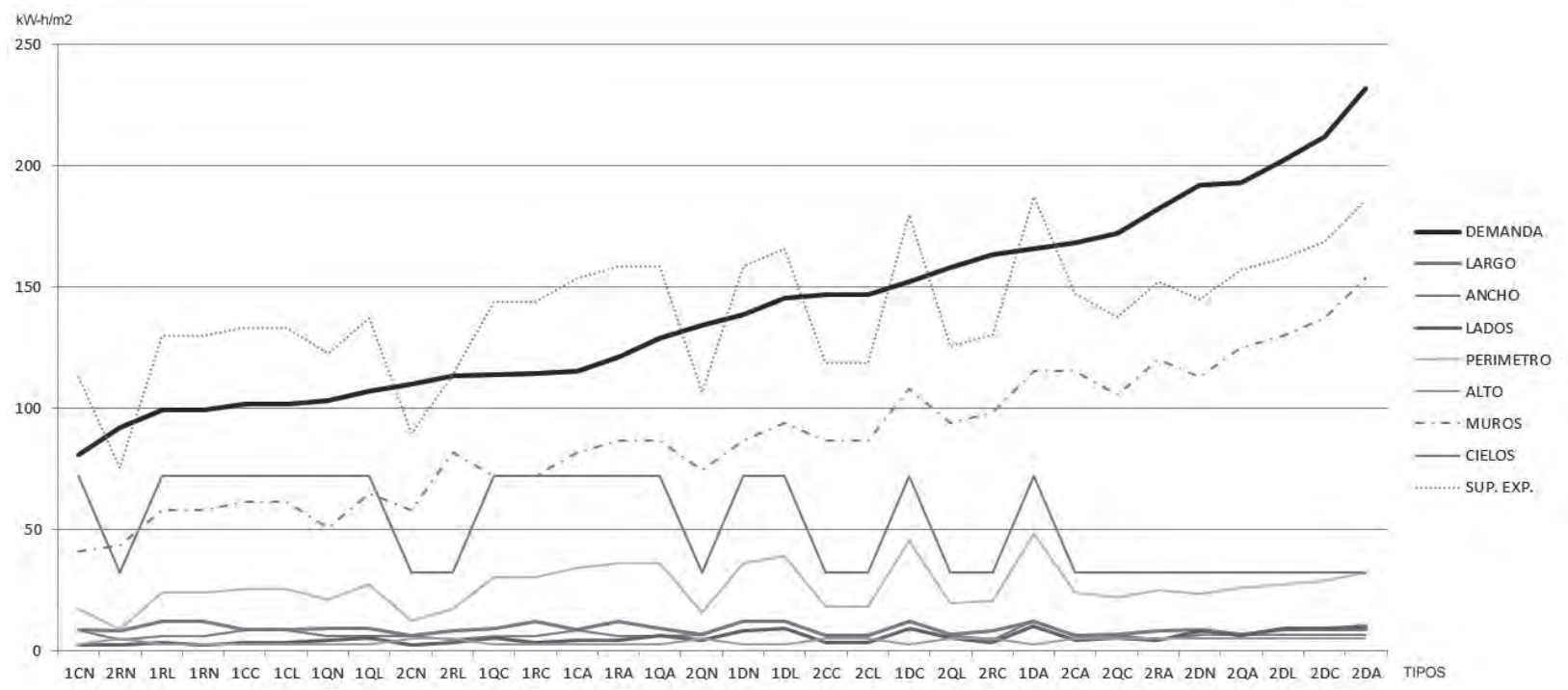

Fuente: Elaboración propia.

que según las referencias estudiadas influyen en el comportamiento ambiental. Se excluyó el sentidode orientación solar, que se reveló con una escasa incidencia, dejando entonces 32 modelos de diferentes niveles, configuración y adosamiento. En las cuales se midió la longitud (mayor dimensión horizontal total), ancho (menor dimensión horizontal total), cantidad de lados expuestos, perímetro (sumatoria de longitudes de cada cara vertical expuesta), altura total, y se calcularon relaciones entre éstos valores; proporción entre longitud y ancho total, superficie de muros expuestos (perímetro por altura) y superficie completa expuesta (adicionando cielos). Los cuales se expresan luego en proporción respecto a la superficie en planta y al volumen climatizado (en estos casos $72 \mathrm{~m}^{2}$ de superficie y $172,8 \mathrm{~m}^{3}$ de volumen), para generalizarlos como indicadores para edificaciones similares, de acuerdo a lo sugerido en estudios previos (tabla 3). En los cuales se puede advertir que oscilan variablemente (excepto altura 


\section{FIGURA 9. GRÁFICO DE RELACIONES GEOMÉTRICAS SEGÚN CASOS ORDENADOS POR DEMANDA.}

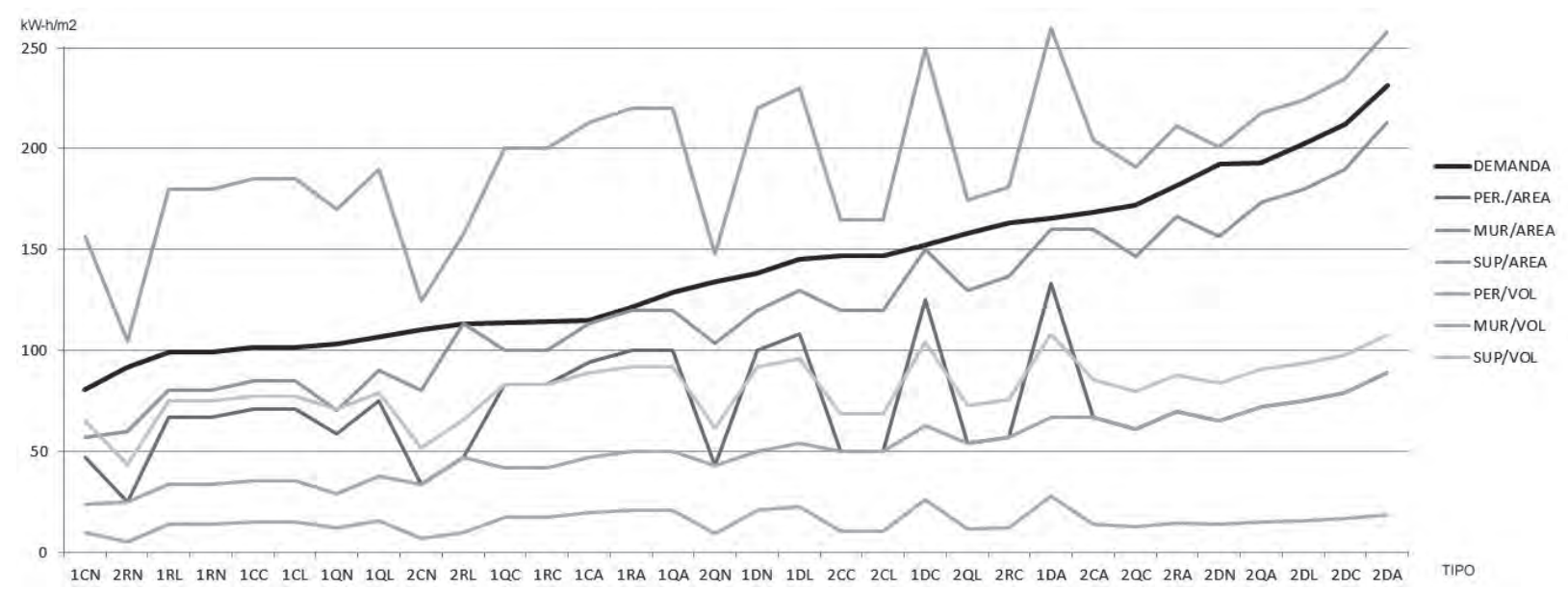

Fuente: Elaboración propia.

y cielos que dependen sólo de poseer uno o dos niveles), y la relación de superficie expuesta/volumen, que se conoce generalmente como "factor de forma", varía desde 0,44 hasta 1,08 en cierta equivalencia con la demanda (aunque estos extremos no corresponden precisamente al valor más bajo o más alto respectivamente).

Disponiendo las modelos de acuerdo a su magnitud de demanda (el valor medio según los resultados entre cada orientación), se advierte la progresión de los requerimientos energéticos según los indicadores geométricos (figura 8).
Las mediciones básicas presentan un desarrollo parejo, en que la cantidad de lados, altura y perímetro evidencian una mayor correlación con la demanda, lo mismo que las proporciones más generales (que en el gráfico se han multiplicado por cien para expresarlas comparativamente), aunque con relevantes oscilaciones. De hecho la superficie total expuesta, que es el valor más mencionado en las referencias como "factor de forma", presenta un crecimiento equivalente a la demanda, pero en menor magnitud, y con variaciones sustanciales. Revelando que, al menos en 


\section{FIGURA 10. GRÁFICO DE SUPERFICIE DE MUROS EXPUESTOS EN RELACIÓN A LAS DEMANDAS ENERGÉTICAS.}

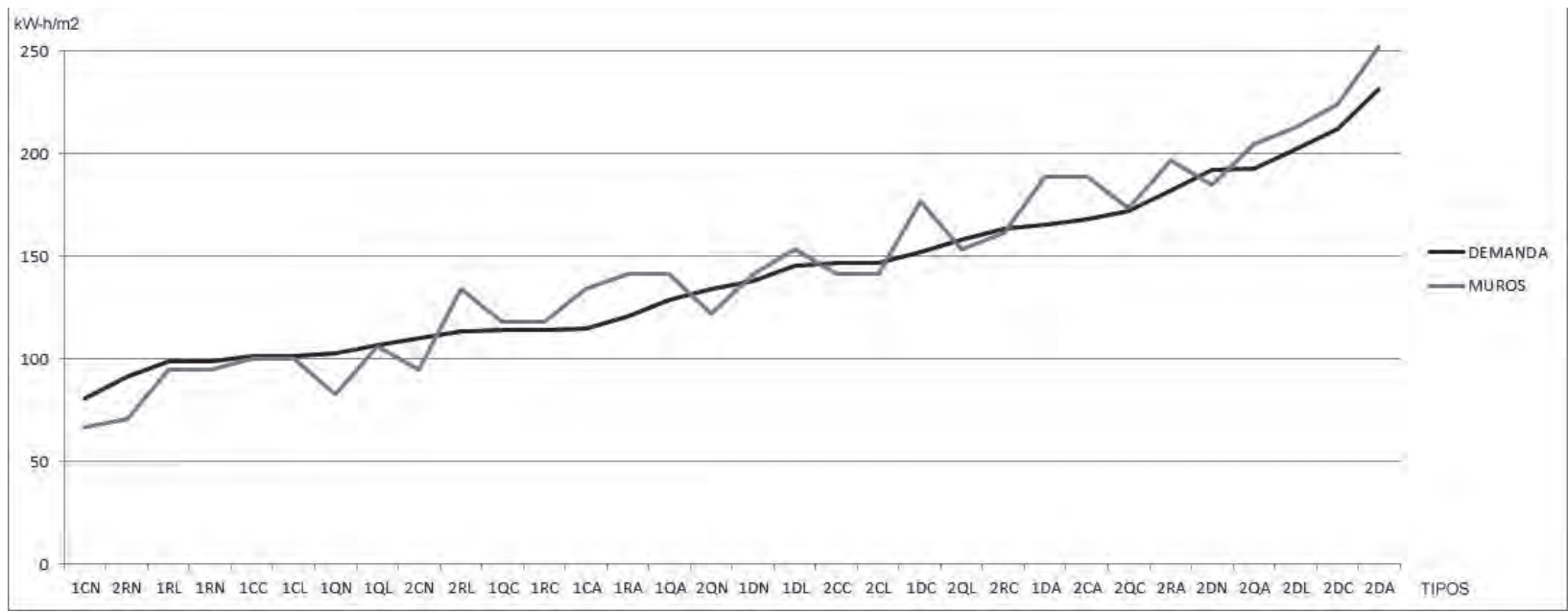

Fuente: Elaboración propia.

estas construcciones, es expresivo de sus requerimientos energéticos, pero no de una manera cabalmente directa.

El valor más equivalente a la demanda en estos casos corresponde a la superficie total de muros expuestos (perímetro por altura), y para obtener una progresión equivalente basta con multiplicar por 1,64 y se obtiene una similaridad media general del 2,5\% (figura 10). Esto quiere decir que en viviendas de estas características materiales y superficie de $72 \mathrm{~m}^{2}$, por cada metro cuadrado de muro al exterior la demanda energética se incrementa en 1,64 kW-h al año, o por cada metro de longitud en su perímetro externo, esto aumenta 4 kW-h o 8 kW-h según sea de uno o dos pisos. Esta magnitud puede parecer leve con respecto al costo directo del kW (aprox. USD 0,25 en Chile), pero si se considera su incidencia total puede duplicar la demanda de una forma más regular. Lo que puede corresponder al doble del gasto doméstico, y a un monto similar a la construcción de la vivienda en la mitad de su vida útil 
(25 años). Es decir, una casa de forma más enrevesada, separada y/o baja puede implicar para sus ocupantes el doble del costo en el tiempo, o poseer una condición ambiental inferior si no son capaces de financiarlo. Además del impacto ambiental y social que produce el incremento del gasto energético.

Revisar la extensión de muros exteriores en las edificaciones residenciales en la zona, considerándolo proporcional al área o el volumen, podría sugerir su demanda energética potencial, y relación de gasto o confort estimado. Motivando una modificación formal que reduzca sus requerimientos ambientales en el diseño inicial, sin deteriorar su capacidad funcional o expresión arquitectónica, que se puede lograr igualmente de manera adecuada en configuraciones compactas. Fomentando un control del gasto doméstico y del impacto ecológico global, además de la mantención de un adecuado confort interior y calidad ambiental, a través de acciones que no implican mayores inversiones ni la incorporación de materialidades o equipos adicionales.

Este indicador podría implementarse en nuevos proyectos o adaptaciones de existentes, por ejemplo relacionado con una rebaja impositiva para promover edificaciones más eficientes. Como también como referencia en estrategias de diseño, operaciones comerciales o programas de mejoramiento, para estimar sus condiciones operativas permanentes o aplicar correcciones significativas.
Promoviendo construcciones más concentradas y ampliaciones que completen los volúmenes construidos. Este indicador es particularmente relevante por cuanto no se revisa en los procesos regulares de edificación residencial ni tampoco en las acciones o certificaciones de eficiencia energética (que usualmente comparan edificaciones de similar forma). A la vez que las tendencias sociales y arquitectónicas se orientan precisamente a aumentar esta condición, motivando viviendas de mayor independencia y sofisticación, además del progresivo incremento en requerimientos residenciales, provocando de este modo, un aumento más sustancial en los consumos energéticos y una sobrecarga en las medidas de mitigación. En ese sentido expresar esta relación es relevante para que el diseño, planeación y gestión habitacional contribuya a una mejor calidad ambiental.

\section{Conclusiones}

Este trabajo revisa incidencias de la forma geométrica en el desempeño energético de los edificios según diversos documentos, en particular referidas a las viviendas unifamiliares en el centro-sur de Chile. Analizando las condiciones indicadas en estudios de la zona, estadísticas y en un análisis de cincuenta viviendas existentes. Se ha efectuado además simulaciones paramétricas de un centenar de casos representativos de las principales variaciones con un mismo modelo de vivienda, que han 
revelado influencias relevantes y consistentes con una composición material y localización determinada. Destacando la incidencia de la diversidad morfológica, contigüidad y altura, en relación a una menor demanda energética, y por ende mejor calidad ambiental. Se sugiere en este caso un factor de regulación a través de la longitud y altura de muros exteriores, como el indicador más expresivo de estas condiciones formales (fundamentalmente debido a las características materiales prevalecientes), con el fin de proponer consideraciones volumétricas paraviviendas nuevas en la zona, de manera complementaria a otras medidas. Promoviendo un diseño residencial más eficiente y confortable, a través de moderar la extensión de los muros y mejorar su composición térmica.

Se deben revisar otras configuraciones constructivas, materialidades distintas, estrategias pasivas (como modificar el diseño de aperturas), patrones de ocupación diferentes o equipamiento, así como de diferentes superficies o detalles de las viviendas. Sin embargo, estos aspectos presentan una influencia similar a otros estudios, por tanto, sería esperable una relación equivalente, aunque de magnitudes distintas. Del mismo modo, se puede revisar la aplicación de estas condiciones en otros contextos o tipologías, especialmente en climas más cálidos, en edificaciones mayores o configuraciones radicalmente distintas, en que igualmente este estudio puede constituir una referencia sobre el proceso de trabajo.
La demostración de influencias formales en el comportamiento ambiental de viviendas unifamiliares según la configuración y ubicación estudiada es particularmente relevante, porque sugiere una reducción en las demandas energéticas, menores gastos domésticos y/o mejoramiento del confort interior. Con escasa inversión en relación a otras acciones de constructivas que usualmente implican gastos adicionales (incrementar aislación, cambiar ventanas, instalar equipos) y poseen menores incidencias. De modo que en configuraciones similares para esta zona se puede promover una menor magnitud de muros exteriores, así como un mejoramiento material, sin deteriorar la expresión arquitectónica, para reducir los requerimientos ambientales e incrementar su calidad residencial. Sugiriendo consideraciones de diseño habitacional que contribuyen a la habitabilidad y sostenibilidad, así como a la diversidad arquitectónica y social.

\section{Bibliografía}

ALBACITI, Rossano y PASSERINI, Francesco. Energy efficiency, building shape and heating requirements: a parametric approach in Italian conditions. [En línea]. Central Europe towards Sustainable Building. 2010. Disponible en: http://cesb.cz/ cesbl0/papers/2_energy/111.pdf

AMBIENTE Consultores Programa de inversión pública para fomentar el reacondicionamiento térmico del parque construido de viviendas, 
587-368-LE06. Santiago, Chile, MIMVU. 2007, $19 \mathrm{p}$.

ASHRAE. IWEC2, Weather Data. (En linea). ASHRAE'S IWEC2 weather files for international locations. S.f. Disponible en: http://weather.whiteboxtechnologies.com/ASHRAE/home.

BUSTAMANTE, Waldo. Guía de diseño para la eficiencia energética en la vivienda social. Santiago, Ministerio de Vivienda y Urbanismo División Técnica de Estudio y Fomento Habitacional y Programa País de Eficiencia Energética (CNE). 2009. 216 p.

CAPOZZOLI, Alfonso y CORRADO, Vincenzo. Impacts of architectural design choice son building energy performance applications of uncertainty and sensitivity techniques. [En línea]. International Building Performance Simulation Association. 2009. Disponible en: http://www.ibpsa.org/proceedings/ BS2009/BS09_1000_1007.pdf

CDT Corporación de Desarrollo Tecnológico. Estudio de usos finales y curva de oferta de la conservación de la energía en el sector residencial. Cámara Chilena de la Construcción, Santiago. 2010.

CELIS, Flavio, GARCÍA, Rodrigo, TREBILCOCK, Maureen, ESCORCIA, Olavo, BRUSCATO, Underléa y DIAZ, Muriel. Análisis energético de las viviendas del centro-sur de Chile.[En línea]. Arquiteturarevista. 8(1): 62-75, 2012.ISSN: 1808-5741. Disponible en: http://revistas.unisinos.br/index.php/ arquitetura/article/view/arq.2012.81.07

CEN Comité Européen de Normalisation. Energy performance of buildings - Methods for expressing energy performance and for energy certification of buildings. Brussels, CEN. 2005.

CHOIA, In Young, CHOA, SungHeui y KIM, Jeong Tai. Energy consumption characteristics of highrise apartment buildings according to building shape and mixed-use development. [En línea]. Energy and Buildings. 46: 123-131, March 2012. ISSN 0378-7788. Disponible en: http://dx.doi. org/10.1016/j.enbuild.2011.10.038

CNE Comisión Nacional de Energía. Irradiancia solar en territorios de la República de Chile: registro solarimétrico. Santiago, CNE/PNUD/UTFSM. 2008.

DANIELSKI, Itai. Energy variations in apartment buildings due to different shapefactors and relative size of common áreas. En: World Renewable Energy Congress (2011, Linköping, Sweden).

DEPECKER, Patrick, MENEZO,Christophe, VIRGONE, Joseph y LEPERS, Stephane. Design of building shape and energy consumption. [En línea]. Building and Environment. 36(5): 627-635, June 2001. ISSN 0360-1323. Disponible en: http://dx.doi. org/10.1016/S0360-1323(00)00044-5

FISSORE, Adelqui Sch. La realidad energética en el sector residencial de la región del Bío-Bío. (En linea). Energy and Climate Partnership of the Americas (ECPA). 2012. (Fecha de consulta: 11 junio 2012). Disponible en: http://www.ecpamericas.org/ data/files/initiatives/energy_efficiency_working_ group/eewg_chile_workshop_mission_2012/ Presenta-AFS-ECPA.pdf 
GARCíA, Pilar y BROXFORD, Ben. Policies to reduce residential energy consumption in Región Metropolitana of Chile, by socio-economic status and home type.[En línea]. Revista Hábitat Sustentable. 2(2): 2-18, 2012. ISSN 0719-0700. Disponible en: http://www.revistahabitatsustentable.cl/ojs/index.php/arquitec/article/ view/434

HATT, Tobias. El estándar passivhaus en el centro-sur de Chile: un estudio paramétrico multifactorial. [En línea]. Chile, Universidad del Bío-Bío. 2012. Tesis doctoral en arquitectura y urbanismo. Disponible en: http://cybertesis.ubiobio.cl/tesis/2012/hatt_t/doc/hatt_t.pdf

INE Instituto Nacional de Estadísticas. XVII Censo nacional de población y de vivienda, 2002. [En línea].INE. 2002. [Fecha de consulta: 11 Junio 2012]. Disponible en: http://www.ine. cl/canales/usuarios/cedoc_online/censos/pdf/ censo_2002_volumen_II.pdf

INN Instituto Nacional de Normalización. Norma chilena oficial Nch 853.Of91 acondicionamiento térmico - envolvente térmica de edificios - cálculo de resistencias y transmitancias térmicas. Santiago de Chile, INN. 2007.

- Norma chilena oficial Nch 1960.Of.89 aislación térmica - cálculo de coeficientes volumétricos globales de pérdidas térmicas. Santiago de Chile, INN. 1989.

- Norma chilena oficial Nch 1079 arquitectura y construcción, zonificación climática-habitacional y recomendaciones de diseño arquitectónico. Santiago de Chile, INN. 2008.

JIJI, Latif M. Heatconduction Berlin, Springer. 2009. ISBN 978-3-642-01266-2. 431 p.

MEC Ministerio de Energía, División de Prospectiva y Política Energética. Balance nacional de energía 2011. [En línea]. Balance Energético - Ministerio de Energía. 2012. [Fecha de consulta: 22 febrero 2013]. Disponible en: http://www.minenergia.cl/ archivos_bajar/bne/BNE2011.xls

MINVU Ministerio de Vivienda y Urbanismo. Informativo estadístico de edificación. (18), diciembre 2012.

NEUFERT, Ernst. Arte de proyectar en arquitectura: fundamentos, normas y prescripciones sobre construcción, dimensiones de edificios, locales y utensilios, instalaciones, distribución y programas de necesidades. México. G. Gili. 1995. ISBN 968-6085-88-2.

OLGYAY, Victor. Arquitectura y clima: manual de diseño bioclimático para arquitectos y urbanistas. Barcelona, G. Gili. 1998. 203 p. ISBN 84-252-1488-2.

OURGHI, Ramzi, AL-ANZI, Adnan y KRARTI, Moncef. A simplified analysis method to predict the impact of shape on annual energy use for office buildings. [En línea]. Energy Conversion and Management. 48(1): 300-305, January 2007. ISSN 01968904. Disponible en: http://dx.doi.org/10.1016/j. enconman.2006.04.011

PACHECO R., Ordónez, J. y MARTÍNEZ, G. Energy efficient design of building: A review.[En línea]. 
Renewable and Sustainable Energy Reviews. 16(6): 3559-3573, 2012. ISSN 1364-0321. Disponible en:http://dx.doi.org/10.1016/j.rser.2012.03.045

PARASONIS, Josifas, KEIZIKAS, Andrius, ENDRIUKAITYTÚ, Audroné y KALIBATIENÚ, Diana. Architectural solutions to increase the energy efficiency of buildings. [En línea]. Journal of Civil Engineering and Management. 18(1): 71-80, 2012. ISSN 18223605. Disponible en: http://dx.doi.org/10.3846/1 3923730.2011 .652983

RUANO, Miguel. Un Vitruvio ecológico: principios y práctica del proyecto arquitectónico sostenible. Barcelona, G. Gili. 2008. 159 p. ISBN 978-84-252-2155-2.

SARMIENTO, Pedro y HORMAZABAL, Nina. Habitabilidad térmica en las viviendas básicas de la zona central de Chile, a la luz de los resultados preliminares del proyecto Fondef D00I103941. [En línea]. Revista INVI. 18(46): 23-32, 2003. ISSN 0718-8358. Disponible en: http://www. revistainvi.uchile.cl/index.php/INVI/article/ view/400/834

SERRA, Rafael y $\mathrm{COCH}$, Helena. Arquitectura y energía natural. Barcelona, Universitat Politècnica de Catalunya. 1995. 395 p. ISBN 84-7653-505-8.

TUHUS-DUBROW, Daniel y KRARTI,Moncef. Geneticalgorithm based approach to optimize building envelope design for residential buildings. [En línea]. Building and Environment. 45(7): 1574-1581, July 2010. ISSN: 0360-1323. Disponible en: http:// dx.doi.org/10.1016/j.buildenv.2010.01.005 